\title{
Structural and chemical analysis of mixed cation antiferromagnetic layered metal chalcophosphate $\mathrm{FeCoP}_{2} \mathrm{~S}_{6}$
}

\author{
Matthew Cheng ${ }^{1}$, Yea-Shine Lee ${ }^{2}$, Roberto dos Reis ${ }^{2}$, Abishek Iyer $^{3}$, Daniel Chica ${ }^{3}$, Mercouri Kanatzidis ${ }^{3}$ \\ and Vinayak Dravid ${ }^{2}$ \\ ${ }^{1}$ Department of Materials Science and Engineering, Northwestern University, EVANSTON, Illinois, United \\ States, ${ }^{2}$ Department of Materials Science and Engineering, Northwestern University, United States, \\ ${ }^{3}$ Department of Chemistry, Northwestern University, United States
}

Since the discovery of graphene by Geim and Novoselov in 2004[1], research interest in two dimensional (2D) materials have risen exponentially in the following decades. Great emphasis is placed on the discovery and utilization of new 2D systems that can be used to expand the library of 2D materials for use in next generation devices and applications that goes beyond that of conventional semiconductors. In particular, 2D ferroic materials are of great interest as potential solutions for high density data storage and nonvolatile memory that do not suffer from the constraints of conventional magnetic materials.

The metal chalcophosphates are a family of $2 \mathrm{D}$ materials that adopts a stoichiometry of $\mathrm{M}^{2+}{ }_{2} \mathrm{P}_{2} \mathrm{X}_{6}$ or $\mathrm{M}^{1+} \mathrm{M}^{3+} \mathrm{P}_{2} \mathrm{X}_{6}$. The three atom thick monolayers are formed from two layers of chalcogen atoms sandwiching a middle layer of transition metal cations and a phosphorus dimer which occupies octahedral sites in respect with the chalcogen atoms (Figure 1a). As these layers are bound by van der Waals interactions, which allows for exfoliation to the monolayer limit.

The magnetic structures of many members of this family of materials, such as $\mathrm{Fe}_{2} \mathrm{P}_{2} \mathrm{~S}_{6}$ and $\mathrm{Ni}_{2} \mathrm{P}_{2} \mathrm{~S}_{6}$, have been studied at length. However, even though all the magnetic chalcophosphates have been found to order with an antiferromagnetic coordination when cooled beneath the Neels temperature the precise details of their magnetic structures are dependent on the choice of the of the transition metal cations that is included in the octahedral sites[2]. For example, the magnetic moments of $\mathrm{Fe}_{2} \mathrm{P}_{2} \mathrm{~S}_{6}$ are best described as Ising-type with the magnetic moments pointing out of the planes of stacking[3] while $\mathrm{Ni}_{2} \mathrm{P}_{2} \mathrm{~S}_{6}$ is arranged in an XY or XXZ orientation in plane[4]. This complexity is further increased when a second cation species is introduced into the lattice as clustering of atoms into specific sites may become apparent[5]. It has been found that $\mathrm{FeNiP}_{2} \mathrm{~S}_{6}$ exhibits spin frustration but is best classified as a magnetic glass[6] while $\mathrm{FeMnP}_{2} \mathrm{~S}_{6}$ is reported to be a spin glass[7]. One may notice that these studies were carried out with elements that are close to Fe on the periodic table of elements except for Co. Thus, it is logical to explore and report on the structure-property relationships of a mixed metallic system with Fe that incorporates Co which exhibits a much closer Neels temperature in the form of $\mathrm{Co}_{2} \mathrm{P}_{2} \mathrm{~S}_{6}$ at $120^{\circ} \mathrm{K}$ and $\mathrm{Fe}_{2} \mathrm{P}_{2} \mathrm{~S}_{6}$ at $123^{\circ} \mathrm{K}$.

In this study, we report on the structural characterization of mixed metal cation $\mathrm{FeCoP}_{2} \mathrm{~S}_{6}$ prepared through a novel flux method[8]. The powder X-ray diffraction (PXRD) spectra for $\mathrm{Fe}_{2} \mathrm{P}_{2} \mathrm{~S}_{6}$ and $\mathrm{Co}_{2} \mathrm{P}_{2} \mathrm{~S}_{6}$ exhibits good agreement with the simulated diffraction patterns of each species and the PXRD spectra for $\mathrm{FeCoP}_{2} \mathrm{~S}_{6}$ follows Vegard's Law (Figure 1c). Scanning electron microscopy X-ray energy dispersive spectroscopy was used to corroborate the stoichiometry of the material which is shown in Figure $1 \mathrm{~d}$. The compositions of each sample are in reasonable agreement with the expected stoichiometry. Figure 2 illustrates the initial TEM analysis of the sample. Low magnification TEM images were taken with accompanying selected area electron diffraction (SAED) patterns (Figure 2a-c). The SAED patterns indicate the presence of $120^{\circ}$ rotational twins along the layer of stacking (Figure 2d-f). High resolution TEM images show either the $\{-331\}$ or $\{060\}$ planes as this are dependent on the rotational twin boundaries present in the sample (Figure $2 \mathrm{~g}$-i). Finally, core-loss electron energy loss spectrum (EELS) for $\mathrm{Fe}_{2} \mathrm{P}_{2} \mathrm{~S}_{6}, \mathrm{FeCoP}_{2} \mathrm{~S}_{6}$, and $\mathrm{Co}_{2} \mathrm{P}_{2} \mathrm{~S}_{6}$ is acquired (Figure $2 \mathrm{j}$ ). The valence state of the monometallic and bimetallic systems are then qualitatively considered using the respective $\mathrm{Fe} \mathrm{L}_{2}$ and $\mathrm{L}_{3}$ edges and the $\mathrm{Co} \mathrm{L}_{2}$ and $\mathrm{L}_{3}$ edges. It was found that the $\mathrm{L}_{3} / \mathrm{L}_{2}$ ratio for both $\mathrm{Fe}$ and $\mathrm{Co}$ are reduced when considering the bimetallic system in comparison with the monometallic systems. In $\mathrm{Fe}_{2} \mathrm{P}_{2} \mathrm{~S}_{6}$, the $\mathrm{Fe} \mathrm{L}_{3} / \mathrm{L}_{2}$ is $\sim 2.5$ while in $\mathrm{FeCoP}_{2} \mathrm{~S}_{6} \mathrm{Fe} \mathrm{L}_{3} / \mathrm{L}_{2}$ is $\sim 0.8$. In comparison, in $\mathrm{Co}_{2} \mathrm{P}_{2} \mathrm{~S}_{6}$ the $\mathrm{Co} \mathrm{L}_{3} / \mathrm{L}_{2}$ is 
$\sim 2.1$ while $\mathrm{Co}_{3} / \mathrm{L}_{2}$ in $\mathrm{FeCoP}_{2} \mathrm{~S}_{6}$ is $\sim 1.3$. As has been demonstrated in oxide systems the larger the $\mathrm{L}_{3} / \mathrm{L}_{2}$ ratio is the lower the valence state of the specific metal cation in consideration[9]. This consistent reduction in the $\mathrm{L}_{3} / \mathrm{L}_{2}$ could be due to a mixing of valence states between the $2+$ and $3+$ states for both $\mathrm{Fe}$ and Co in the bimetallic the $\mathrm{FeCoP}_{2} \mathrm{~S}_{6}$ system. The presentation will reveal, in depth, the nature of the cation distribution in bimetallic $\mathrm{FeCoP}_{2} \mathrm{~S}_{6}$ and the resulting impact on its electronic structure and magnetic properties in comparison with that of monometallic $\mathrm{Fe}_{2} \mathrm{P}_{2} \mathrm{~S}_{6}$ and $\mathrm{Co}_{2} \mathrm{P}_{2} \mathrm{~S}_{6}$. We believe that these metal chalcophosphates will prove to be an interesting addition to the 2D library of materials due to their intrinsic ferroic properties that warrants further study and exploration.

\section{Acknowledgements}

Funding provided by the NSF (DMR-1929356) and the ARO (W911NF1910335). This work made use of the EPIC facility of Northwestern University's NUANCE Center, which has received support from the SHyNE Resource (NSF ECCS-2025633), the IIN, and Northwestern's MRSEC program (NSF DMR-1720139).

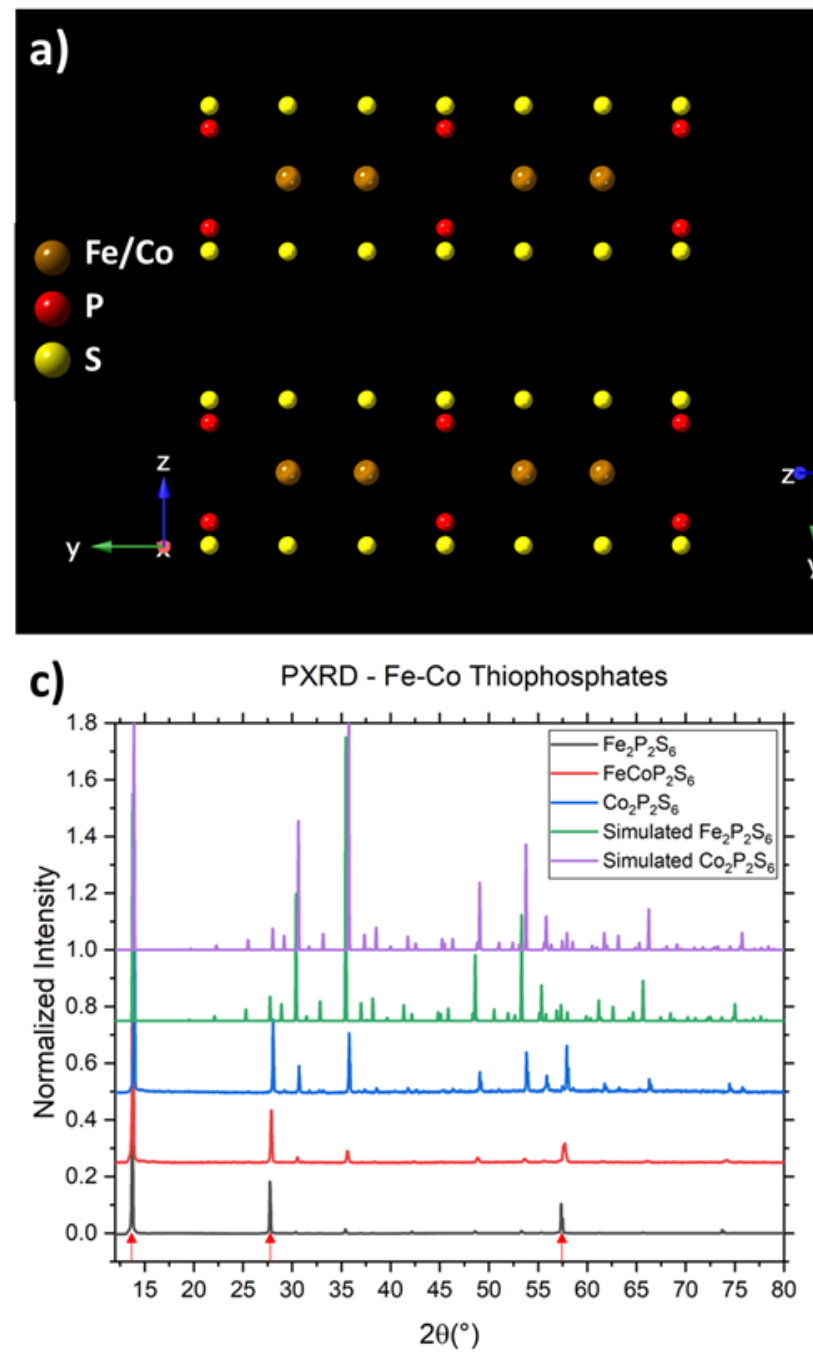

\section{b)}

d)

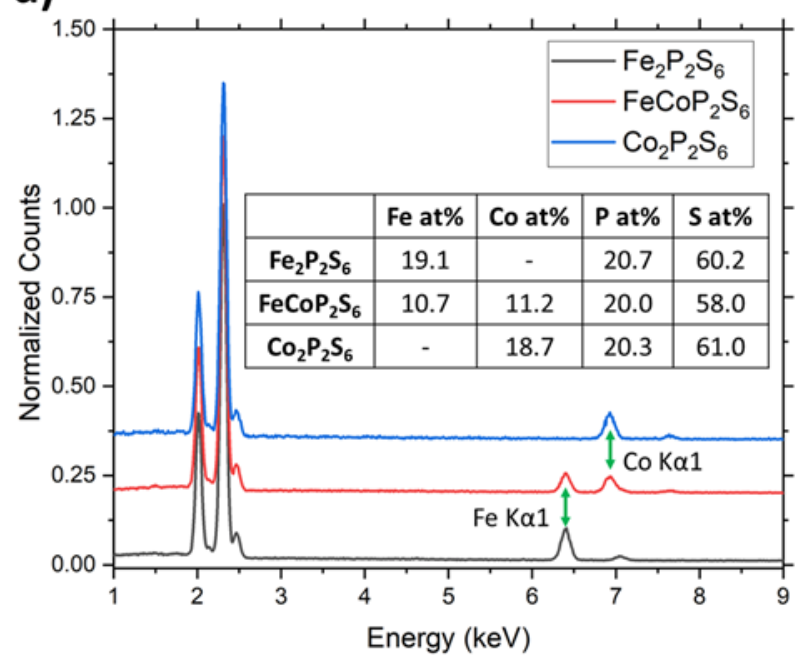

Figure 1. a) Side-view along the a-axis of iron(cobalt) thiophosphate. b) View along $c^{*}$-axis orthogonal to the planes of stacking of iron (cobalt) thiophosphate. c) Powder X-Ray Diffraction (PXRD) of Fe2P2S6, FeCoP2S6, and Co2P2S6. The (001)-planes are indicated with red arrows. d) SEM-EDS of Fe2P2S6, FeCoP2S6, and Co2P2S6. Inset table showing the atomic percent of Fe, Co, P, and $\mathrm{S}$ for each

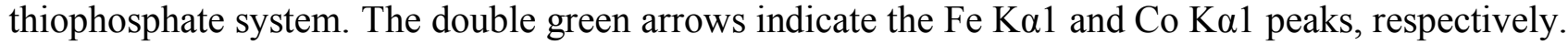



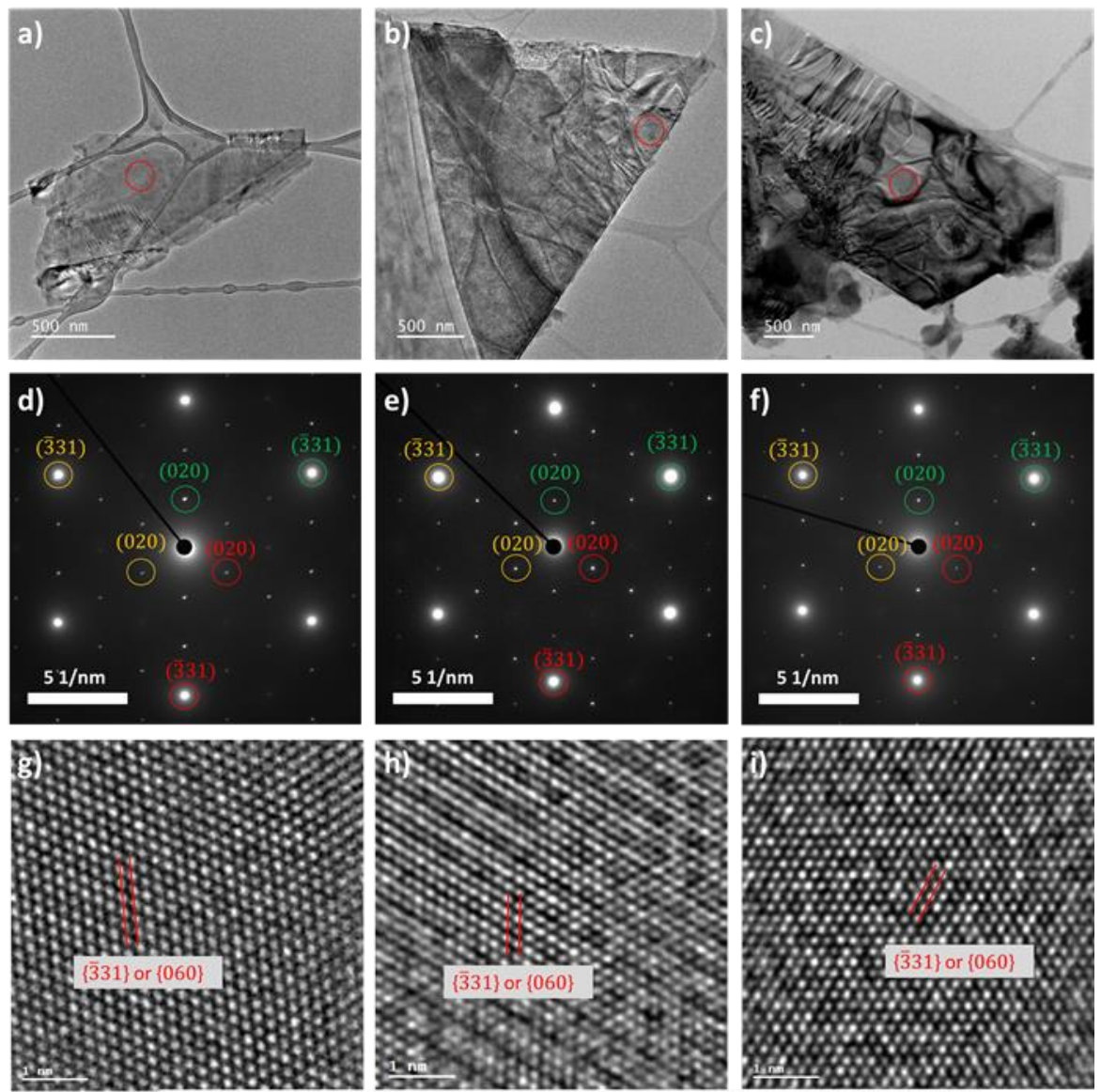

j)

EELS - Fe-Co Thiophosphates

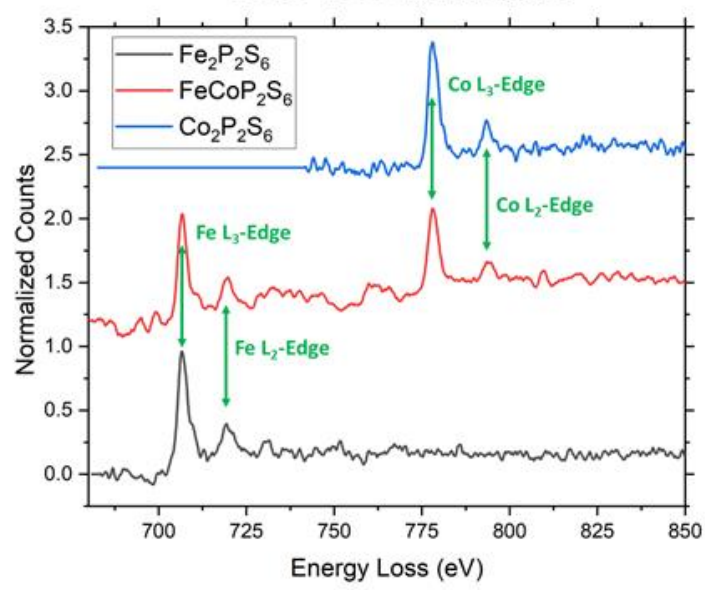

Figure 2. a-c) Low resolution TEM bright-field images for Fe2P2S6, FeCoP2S6, and Co2P2S6, respectively. The red circle indicates the location of the selected area electron diffraction (SAED) aperture where SAED patterns are taken. d-f) SAED patterns for Fe2P2S6, FeCoP2S6, and Co2P2S6, respectively. SAED pattern taken along the $\mathrm{c}^{*}$-axis which is nearly parallel to the -zone axis. The SAED pattern exhibits the presence of $120^{\circ}$-twins along the out-of-plane direction. g-i) High resolution TEM (HRTEM) images of Fe2P2S6, FeCoP2S6, and Co2P2S6, respectively with the $\{-331\}$ or $\{060\}$ planes indicated. j) Coreloss EELS for Fe2P2S6, FeCoP2S6, and Co2P2S6 with the Fe-L Edges and Co-L Edges indicated. The atomic percentage indicating the ratio of $\mathrm{Fe}$ and $\mathrm{Co}$ are shown. 


\section{References}

1. Novoselov, K.S., et al., Electric Field Effect in Atomically Thin Carbon Films. Science, 2004. 306(5696): p. 666.

2. Wang, F., et al., New Frontiers on van der Waals Layered Metal Phosphorous Trichalcogenides. Advanced Functional Materials, 2018. 28(37): p. 1802151.

3. Lee, J.-U., et al., Ising-Type Magnetic Ordering in Atomically Thin FePS3. Nano Letters, 2016. 16(12): p. 7433-7438.

4. Kim, K., et al., Suppression of magnetic ordering in XXZ-type antiferromagnetic monolayer NiPS3. Nature Communications, 2019. 10(1): p. 345.

5. Goossens, D.J., et al., Local order in layered NiPS3and NiO.7MgO.3PS3. Journal of Physics: Condensed Matter, 2011. 23(6): p. 065401.

6. Goossens, D.J., et al., Magnetic structure and glassiness in Fe0.5NiO.5PS3. Journal of Magnetism and Magnetic Materials, 2013. 334: p. 82-86.

7. Graham, J.N., et al., Local nuclear and magnetic order in the two-dimensional spin glass Mn0.5Fe0.5PS3. Physical Review Materials, 2020. 4(8): p. 084401.

8. Chica, D.G., et al., P2S5 Reactive Flux Method for the Rapid Synthesis of Mono-and Bimetallic 2D Thiophosphates M2-xM'xP2S6. Accepted in Inorganic Chemistry, 2021.

9. Wang, Z.L., J.S. Yin, and Y.D. Jiang, EELS analysis of cation valence states and oxygen vacancies in magnetic oxides. Micron, 2000. 31(5): p. 571-580. 